\title{
Fluoride and calcium concentrations in the biofilm fluid after use of fluoridated dentifrices supplemented with polyphosphate salts
}

\author{
Mariana Emi Nagata ${ }^{1}$ Alberto Carlos Botazzo Delbem ${ }^{1} \cdot$ Kevin Bruce Hall $^{1}$. \\ Marília Afonso Rabelo Buzalaf ${ }^{2}$. Juliano Pelim Pessan ${ }^{1,3}$
}

Received: 28 August 2015 / Accepted: 24 April 2016 / Published online: 27 May 2016

(C) Springer-Verlag Berlin Heidelberg 2016

\begin{abstract}
Objectives The present study evaluated fluoride (F) and calcium $(\mathrm{Ca})$ concentrations in the biofilm fluid formed in situ under cariogenic challenge after using $\mathrm{F}$ dentifrices supplemented or not with sodium trimetaphosphate (TMP) or calcium glycerophosphate (CaGP).

Methods Volunteers $(n=12)$ were randomly divided into 5 groups according to the toothpastes used: placebo (without $\mathrm{F}$, CaGP or TMP), 1100 ppm F (1100F) and low-fluoride dentifrice (LFD, $550 \mathrm{ppm} \mathrm{F}$ ) with no supplementation $(550 \mathrm{~F})$ or supplemented with $1 \%$ TMP (550F-TMP) or $0.25 \% \mathrm{CaGP}$ (550F-CaGP). In each phase, volunteers wore palatal appliances containing 4 bovine enamel blocks. Cariogenic challenge was performed with $30 \%$ sucrose solution, 6 times/ day. On the morning of the eigth day, biofilm samples were collected $12 \mathrm{~h}$ and $1 \mathrm{~h}$ after brushing and cariogenic challenge. $\mathrm{F}$ and $\mathrm{Ca}$ analyses in the biofilm fluid were performed with the inverted electrode after buffering with TISAB III and using the Arsenazo III method, respectively. Data were submitted to two-way ANOVA (repeated measures) and Student-NewmanKeuls test $(p<0.05)$.

Results A dose-response relationship was verified between $\mathrm{F}$ concentrations in the dentifrices and in the biofilm fluid. Significant differences were observed among placebo, 550F, and $1100 \mathrm{~F}$ only $1 \mathrm{~h}$ after brushing, without statistical
\end{abstract}

Juliano Pelim Pessan

jpessan@foa.unesp.br

1 Araçatuba Dental School, Univ Estadual Paulista (UNESP), Araçatuba, SP, Brazil

2 Bauru Dental School, University of São Paulo, Bauru, SP, Brazil

3 Department of Pediatric Dentistry and Public Health, Araçatuba Dental School, Univ Estadual Paulista (UNESP), José Bonifácio 1193, Araçatuba, SP 16015-050, Brazil differences among 550F, 550F-TMP, and 550F-CaGP. No defined trend was observed among the groups regarding $\mathrm{Ca}$ concentrations, with the highest values seen for placebo and $550 \mathrm{~F}-\mathrm{CaGP}$

Conclusion The anticaries effect of LFDs supplemented with $\mathrm{CaGP}$ or TMP cannot be related to an increased availability of $\mathrm{F}$ and $\mathrm{Ca}$ in the biofilm fluid.

Clinical significance The better performance of LFDs containing CaGP or TMP shown in previous studies should be attributed to their ability to interact with tooth enamel and with the biofilm, rather to their effect on the biofilm fluid.

Keywords Fluoride · Dentifrice · Biofilm fluid · Sodium trimetaphosphate $\cdot$ Calcium glycerophosphate

\section{Introduction}

The uncertainties surrounding the efficacy of low-fluoride dentifrices (LFD, 500-550 ppm F) against dental caries when compared to conventional dentifrices (CD, 1000 $1100 \mathrm{ppm} \mathrm{F}$ ) has prompted to several studies attempting to increase the anticaries effects of such formulations $[1,2]$. Among the strategies available, the supplementation of LFDs with calcium and/or phosphate salts has been studied in recent years [3]. In vitro and in situ studies showed that LFDs supplemented with calcium glycerophosphate (CaGP) $[4,5]$ or sodium trimetaphosphate (TMP) [6] have a similar anticaries effect when compared to a CD. Such effects were clinically confirmed in a recent randomized trial, in which the progression of caries lesions was shown to be significantly lower in children using the TMP-containing LFD in comparison to the conventional formulation (1100 ppm F), while the progression in the group using a CaGP-containing LFD was similar to the $1100 \mathrm{ppm} \mathrm{F}$ toothpaste [7]. 
The addition of CaGP and TMP to other topically applied fluoridated products has also been shown to promote a synergistic protective effect against dental caries and erosive wear, using in vitro [8-10] and in situ models [4, 5, 11]. Little is known, however, about the mechanisms by which these salts interfere with the de- and remineralization processes of dental enamel. The use of the LFDs supplemented with CaGP or TMP was shown to promote an increase on enamel surface hardness, besides a marked effect on the depth of enamel subsurface lesions [4-6, 12]. Furthermore, F and calcium (Ca) concentrations in biofilm formed in situ in the presence of these toothpastes were significantly higher than their counterparts without calcium or phosphate salts, reaching levels similar to those attained by the use of the CD [4, 13].

Although this increase in biofilm $\mathrm{F}$ and $\mathrm{Ca}$ levels seems to explain the synergistic effect of TMP and CaGP when added to a fluoride dentifrice, the availability of these ions in the fluid phase of the biofilm remains unknown. Previous studies demonstrated that $\mathrm{F}$ and $\mathrm{Ca}$ ions may be retained in biofilm through the formation of mineral deposits [14-16], which may be released from biofilm fluid during a cariogenic challenge. The literature also reports that even when biofilm is not completely removed, $\mathrm{F}$ retained in this reservoir can be released into the fluid of biofilm, reducing demineralization of enamel covered by biofilm [17]. Based on the above, the assessment of the effects of LFDs containing TMP or CaGP on the mineral composition of the biofilm fluid could bring useful information for a better understanding of the mechanisms by which these salts interfere with the dynamics of dental caries.

Thus, the present study aimed to evaluate the concentration of $\mathrm{F}$ and $\mathrm{Ca}$ in the biofilm fluid formed in situ associated with the use of LFDs supplemented with TMP or CaGP, under cariogenic challenge. The study's hypothesis was that the supplementation of LFDs with TMP or CaGP would significantly increase $\mathrm{F}$ levels in the biofilm fluid when compared to their counterpart without TMP or CaGP.

\section{Material and methods}

This study was approved by the Human Research Ethics Committee of Araçatuba Dental School (CAAE 20146313.7.0000.5420) and all participants signed an informed consent form.

The study was carried out through an in situ, double-blind, crossover, and randomized design comprising five experimental phases of 7 days each. A 7-day washout period was done prior to each experimental phase. During this period, the volunteers were instructed to use placebo toothpaste to eliminate possible residual effects from the previous treatments and not use mouthrinse or other topical fluoride agents. Twelve volunteers, regardless of gender, aged 20-34 years-old and resident in Araçatuba participated in the study. The inclusion criteria comprised good general and oral health volunteers, without systemic drugs use that might interfere with biofilm formation or salivary flow. On the other hand, individuals with active caries lesions, who received fluoride applications 2 weeks before the experiment, water activities practitioners, environment polluted by low $\mathrm{pH}$ components workers (industry), smokers, and volunteers diagnosed with systemic diseases (xerostomia, diabetes, autoimmune diseases, malnutrition, gastroesophageal problems) were excluded. The volunteers wore acrylic palatal appliances $(24 \mathrm{~h} /$ day $)$ containing four bovine enamel blocks $(4 \times 4 \times 2 \mathrm{~mm})$ placed $1 \mathrm{~mm}$ below the acrylic level and covered by a plastic mesh to allow dental biofilm accumulation. During each phase, the volunteers dripped a $30 \%$ sucrose solution on the enamel blocks (6 times/day), and brushed their teeth three times a day, for 7 days, using one of the following toothpastes: (1) placebo (fluoride-free), (2) $550 \mathrm{ppm} \mathrm{F}$, (3) $1100 \mathrm{ppm} \mathrm{F}$, (4) 550 ppm F with $0.25 \% \mathrm{CaGP}$, (5) 550 ppm F with $1 \%$ TMP, hereafter abbreviated as placebo, 550F, 1100F, 550FCaGP, and 550F-TMP, respectively. Biofilm samples were collected in the morning of the eigth day after overnight fasting ( 2 enamel blocks), and $60 \mathrm{~min}$ after tooth brushing and exposure to sucrose. A sample of 11 volunteers was calculated based on a previous in situ study conducted with a similar protocol, assessing whole biofilm fluoride concentrations after the use of placebo and $550 \mathrm{ppm} F$ toothpastes (mean difference $=0.16 \mu \mathrm{M} \mathrm{F} / \mathrm{Kg}$, standard deviation $=0.1$ ) [4], considering $\alpha$ - error of $5 \%$ and $\beta$-error of $20 \%$ (SigmaPlot, version 12.0). Assuming a dropout rate of $20 \%$, sample size was then determined as 14 volunteers.

The experimental dentifrices were produced in the laboratory of Pediatric Dentistry from Araçatuba Dental School, using the same basic formulation (except for F, CaGP, and TMP concentrations) with the following components: titanium dioxide, carboxymethyl cellulose, methyl phydroxybenzoate, sodium saccharine, oil peppermint, glycerin, silica abrasive, sodium lauryl sulfate, and water. Formulations without $\mathrm{F}$ (placebo) and containing $\mathrm{F}(\mathrm{NaF}-$ Merck®, Germany) in the concentrations of 550 and $1100 \mathrm{mg} \mathrm{F} / \mathrm{g}$ were obtained. Also, CaGP, or TMP (Sigma®-Aldrich, USA) were added to the 550 ppm F dentifrice at concentrations of 0.25 and $1 \%$, respectively. The CaGP concentration was determined based on studies of Amaral et al. (2013) [4] and Freire et al. (2016) [7], which used in situ and in vivo protocols, respectively. TMP concentration was determined based on the study of Freire et al. (2016) [7]. Fluoride concentrations in the toothpastes were determined using an ion-specific electrode (9409 BN) connected with an ion analyzer (Orion 720 Aplus), previously calibrated with 5 standards $(0.125,0.25,0,5,1.0$, and $2.2 \mathrm{mg} \mathrm{F} / \mathrm{mL}$ ) [18].

Two hundred eighty enamel blocks measuring $4 \times 4 \times 2 \mathrm{~mm}$ were obtained from bovine incisors previously 
stored in $2 \%$ formaldehyde solution ( $\mathrm{pH} 7.0$ ) for 1 month. Enamel blocks were serially polished and selected according to their surface hardness (SH, $369.0 \pm 3.1 \mathrm{KHN}$ ). Each acrylic palatal appliance had 4 enamel blocks, which were randomly assigned into the 5 experimental groups ( $p=0.97$ ). A 4.0-mmdeep space was created in the appliances, leaving $1.0-\mathrm{mm}$ space for dental biofilm accumulation on the enamel blocks. A plastic mesh was fixed in acrylic resin to avoid mechanical disturbance and to induce dental biofilm formation [4].

The cariogenic challenge was produced by the use of a $30 \%$ sucrose solution (Synth, Brazil), which was replaced every $48 \mathrm{~h}$. The volunteers were instructed to remove the appliance from the oral cavity before dropping two drops of sucrose solution on each block (sufficient amount to fill out the space of $1.0 \mathrm{~mm}$ ), 6 times a day, at predetermined times (8:00 am, 11:00 am, 02:00 pm, 05:00 pm, 07:00 pm, 09:00 pm). After dripping, the appliances were left to rest for $5 \mathrm{~min}$ before being returned to the oral cavity, in order to allow the diffusion of the sucrose in the biofilm. Treatment with dentifrices was performed 3 times a day, for 7 days. The volunteers used the transverse technique to standardize the amount of dentifrice and brushed their natural teeth with the device in the oral cavity, therefore producing natural dentifrice/saliva slurry; this was later swished in the oral cavity during $30 \mathrm{~s}$, spat out, and the mouth was gently rinsed with tap water. The volunteers were instructed to use the appliances $24 \mathrm{~h}$ a day and remove only during meals. They were instructed not to use systemic drugs that might interfere with biofilm formation or salivary flow and topical fluoride products throughout the entire experiment.

Biofilm samples were collected in the morning of the eighth day of each experimental phase, at 2 moments (biofilm from 2 enamel blocks each time), with volunteers fasting overnight. The first sample was collected about $12 \mathrm{~h}$ after the last treatment with the toothpastes done on the previous night. Following, the volunteers brushed their teeth with the provided dentifrice and the cariogenic challenge was performed 5 min later. The second sample was then collected $60 \mathrm{~min}$ after tooth brushing. The biofilm was collected with a plastic spatula and weighed in preweighed microcentrifuge cap tubes filled with mineral oil. Tubes were then centrifuged $\left(21,023 g, 5 \mathrm{~min}, 4^{\circ} \mathrm{C}\right)$ in order to separate the biomass from the fluid phase. After centrifugation, a small fraction of the fluid was collected with a micropipette, also filled with mineral oil.

After biofilm fluid separation from its solid phase, it was transferred to the surface of an inverted ion-specific electrode, immersed in mineral oil. Through this microanalysis technique, multiple samples were placed simultaneously on the electrode [19]. The samples were placed on drops of TISAB III (Orion) previously placed on the electrode membrane, in a ratio of 10:1 (sample: TISAB) and were read by the positioning of the reference microelectrode within each sample in order to close the circuit. This electrode was calibrated with standard solutions of known fluoride concentrations (ranging from 1 to $100 \mu \mathrm{M} \mathrm{F})$.

Calcium analyses of biofilm fluid samples were performed by spectrophotometry. A quartz nanopipette of approximately $1 \mu \mathrm{L}$ was used, allowing standardized volumes of calcium standards (ranging from 40 to $200 \mathrm{mM}$ Ca) and samples. Arsenazo III was used as colorimetric reagent [20], and sample readings were performed on a microplate reader (Biotek Eon). Fifty microliters of deionized water and $1 \mu \mathrm{L}$ of the samples/standard were added in each microplate well. Then, $50 \mu \mathrm{L}$ of the colorimetric reagent was added in each plate well, and the plates were shaken during $60 \mathrm{~s}$ in the microplate reader, allowing the reaction between sample and Arsenazo III prior to obtaining the resulting absorbance. The absorbance reader was performed in $610 \mathrm{~nm}$.

Statistical analysis was performed on the software SigmaPlot version 12.0, at a significance level of $5 \%$. Data analysis considered the types of experimental toothpastes and the time of sample collection. Fluoride ( $\log _{10}$ transformed) and calcium (raw) data passed normality (Shapiro-Wilk) and homogeneity tests (Bartlett) and were submitted to two-way, repeated-measures ANOVA, followed by the StudentNewman-Keuls test.

\section{Results}

Table 1 shows mean $\mathrm{F}$ concentrations in the experimental dentifrices, which presented a maximum variation within $10 \%$ according to the allowed for the products.

A dose-response relationship was observed between fluoride concentrations in the dentifrices and the resulting levels in the biofilm fluid $60 \mathrm{~min}$ after the treatment with the toothpastes (Table 2). Significant differences were observed among the dentifrices $(\mathrm{F}=16.7, p<0.001)$ and time after brushing $(\mathrm{F}=38.0, p<0.001)$, with a significant interaction between

Table 1 Mean (SD) concentrations $(\mu \mathrm{g} / \mathrm{g})$ in the experimental fluoride toothpastes

\begin{tabular}{lll}
\hline Dentifrices & \multicolumn{2}{l}{ Concentration $(\mu \mathrm{g} / \mathrm{g})$} \\
\cline { 2 - 3 } & Total fluoride & Ionic fluoride \\
\hline Placebo & $11.6(1.5)$ & $11.1(1.2)$ \\
$550 \mathrm{~F}$ & $521.3(27.7)$ & $550.0(12.6)$ \\
$550 \mathrm{~F}-\mathrm{CaGP}$ & $523.1(21,8)$ & $549.5(5.3)$ \\
$550 \mathrm{~F}-\mathrm{TMP}$ & $553.1(4.4)$ & $558.6(11.3)$ \\
$1100 \mathrm{~F}$ & $1100(48.6)$ & $1119.5(19.6)$ \\
\hline
\end{tabular}


Table 2 Mean (SD) fluoride concentrations $(\mu \mathrm{M})$ in the biofilm fluid formed in situ $12 \mathrm{~h}$ after brushing and $1 \mathrm{~h}$ after brushing and cariogenic challenge (30\% sucrose solution), according to the dentifrices used in each experimental phase

\begin{tabular}{lll}
\hline Dentifrices & \multicolumn{2}{l}{ Time after brushing } \\
\cline { 2 - 3 } & $12 \mathrm{~h}$ & $1 \mathrm{~h}$ \\
\hline Placebo & $5.8(3.1)$ & $5.1^{\mathrm{a}}(3.3)$ \\
$550 \mathrm{~F}$ & $14.5(12.9)$ & $31.2^{\mathrm{b}}(26.4)$ \\
$550 \mathrm{~F}-\mathrm{CaGP}$ & $8.3(6.5)$ & $23.5^{\mathrm{b}}(11.5)$ \\
$550 \mathrm{~F}-\mathrm{TMP}$ & $11.8(7.5)$ & $24.2^{\mathrm{b}}(22.7)$ \\
1100 & $13.5(10.0)$ & $45.4^{\mathrm{c}}(22.8)$ \\
\hline
\end{tabular}

Lowercase superscript letters indicate significant differences among the dentifrices $1 \mathrm{~h}$ after brushing. No significant differences were observed among the groups $12 \mathrm{~h}$ after brushing. Two-way, repeated measures ANOVA (data log transformed), and Student-Newman-Keuls test $(p<0.05), n=12$

the two variables $(\mathrm{F}=4.2, p=0.003)$. For samples obtained $1 \mathrm{~h}$ after brushing (mean $\pm \mathrm{SD}$ ), significant differences were observed among placebo $(5.1 \pm 3.3), 550(31.2 \pm 26.4)$, and $1100 \mathrm{~F}(45.4 \pm 22.8)$ toothpastes, without significant differences among 550F $(31.2 \pm 26.4)$, 550F-CaGP $(23.5 \pm 11.5)$, and 550F-TMP (24.2 \pm 22.7$)$. For samples collected $12 \mathrm{~h}$ after brushing, no significant differences were observed among the dentifrices.

As for $\mathrm{Ca}$ concentrations in the biofilm fluid, significant differences were observed only among the dentifrices $(\mathrm{F}=3.59, p<0.013)$ with no significant differences for time after brushing $(\mathrm{F}=2.37, p<0.151)$ and no interaction between the two variables $(\mathrm{F}=1.90, p=0123)$, as shown in Table 3. No defined trend was observed among the groups, with the highest values seen for placebo and 550F-CaGP. Significant differences were observed among placebo and $1100 \mathrm{~F}$ and placebo and 550F-TMP, with no differences among 550F, 1100F, $550 \mathrm{TMPF}$, and 550FCaGP.

Table 3 Mean (SD) calcium concentrations ( $\mathrm{mM}$ ) in the biofilm fluid formed in situ $12 \mathrm{~h}$ after brushing and $1 \mathrm{~h}$ after brushing and cariogenic challenge (30\% sucrose solution), according to the dentifrices used in each experimental phase

\begin{tabular}{lll}
\hline Dentifrices & \multicolumn{2}{l}{ Time after brushing } \\
\hline & $12 \mathrm{~h}$ & $1 \mathrm{~h}$ \\
Placebo $^{\mathrm{a}}$ & $123.1(63.0)$ & $148.7(58.0)$ \\
$550 \mathrm{~F}^{\mathrm{a}, \mathrm{b}}$ & $112.6(68.5)$ & $93.4(34.0)$ \\
$550 \mathrm{~F}-\mathrm{CaGP}$ & $119.9(42.3)$ & $135.0(34.1)$ \\
$550 \mathrm{~F}-\mathrm{TMP}^{\mathrm{a}}$ & $76.2(31.5)$ & $106.4(27.4)$ \\
$1100^{\mathrm{b}}$ & $86.6(32.3)$ & $100.5(35.4)$ \\
\hline
\end{tabular}

Lowercase superscript letters indicate significant differences among the dentifrices. Two-way, repeated measures ANOVA, and StudentNewman-Keuls test $(p<0.05), n=12$

\section{Discussion}

The addition of calcium and/or phosphate salts to fluoridated toothpastes has been proposed to reduce $\mathrm{F}$ concentration in the products without compromising their anticaries effect when compared to a conventional dentifrice, in order to minimize $\mathrm{F}$ intake from this source and consequently reducing the risk of dental fluorosis. There is considerable in vitro and in situ evidence on the effects of dental products supplemented with TMP $[6,8,21]$ and CaGP $[4,5,12]$ on the dynamics of dental caries using different methods, but information about the mechanism of action of these compounds is still lacking. The present study showed that the addition of TMP or CaGP to low-fluoride toothpastes did not increase the availability of $\mathrm{F}$ and $\mathrm{Ca}$ in the fluid phase of biofilms formed in situ under cariogenic challenge, leading to the rejection of the study's hypothesis.

Literature has shown that the supplementation of lowfluoride toothpastes with TMP or CaGP has a marked effect on enamel hardness, leading to a higher degree of surface hardness and decreased loss of integrated subsurface hardness. The use of a 500- $\mu \mathrm{g}$ F/g toothpaste associated with TMP at concentrations higher than $0.5 \%$ increased surface hardness and decreased $\triangle \mathrm{KHN}$ when compared to the negative control, with the greatest effect observed for the low-F toothpaste containing $3 \%$ TMP (190\% higher) [6]. As for CaGP, the addition of $0.25 \%$ of this salt to a low-fluoride dentifrice decreased mineral loss by $132 \%$, reaching greater protective effect than that seen for the positive control in vitro [12]. The same toothpaste resulted in a $44 \%$ decrease in enamel surface hardness change in situ in comparison with its counterpart without CaGP, having a similar effect than a 1100 ppm F toothpaste [4].

The information above is in line with a mechanism recently proposed, according to which TMP seems to act as a partial barrier to acids, by binding to enamel and forming a "network" able to retain fluoride compounds that are released during subsequent cariogenic challenges [10]. The authors also demonstrated that while the effects of TMP alone are negligible, it has a synergistic effect with fluoride, which gives support to the above-mentioned hypothesis. The same trend was later confirmed by another investigation showing that gels containing $1 \% \mathrm{NaF}$ and $5 \%$ TMP were able to inhibit enamel demineralization similarly to a $2 \% \mathrm{NaF}$ gel, while TMP alone did not reduce demineralization [8]. Regarding CaGP, the possible anticaries mechanism has been suggested to be related to interactions with enamel during the de/remineralizing process in a similar pattern as TMP $[4,5,12]$. It has been also suggested that the availability of $\mathrm{Ca}$ and $\mathrm{F}$ in the enamel from the fluoridated dentifrices associated with $\mathrm{CaGP}$ was the main factor to improve the ability of remineralization [5].

Despite the growing body of evidence on the synergistic effects of fluoride and TMP or CaGP when added to 
toothpastes on enamel hardness composition, little is known about the effects of these salts on the dental biofilm. The use of low-fluoride formulations containing these salts was shown to significantly reduce the formation of extracellular polysaccharides, as well as to significantly raise $\mathrm{F}$ and $\mathrm{Ca}$ concentrations in biofilms formed in situ, in comparison to the $550 \mathrm{ppm} \mathrm{F}$ without TMP [13] or CaGP [4]. These positive results led to the assumption that the increased levels of $\mathrm{F}$ and $\mathrm{Ca}$ in the biofilm would be reflected in a higher availability of these ions in the fluid phase of the biofilm, but such effect was not confirmed in the present study. Although significant differences were seen in $\mathrm{F}$ levels in the biofilm fluid $1 \mathrm{~h}$ after brushing with placebo, $550 \mathrm{~F}$, and $1100 \mathrm{~F}$ dentifrices (what validates the method used), no significant differences were observed among toothpastes containing $550 \mathrm{~F}$, regardless the addition or not of CaGP or TMP. These findings are in line with previous data on the effects of CaGP when added to a conventional dentifrice $(1500 \mu \mathrm{g} \mathrm{F} / \mathrm{g})$, showing no significant differences between toothpastes with or without CaGP at $0.13 \%$ regarding $\mathrm{F}, \mathrm{Ca}$, and inorganic $\mathrm{P}$ concentrations in the biofilm fluid [22]. Although the literature reports an increase in the total biofilm mineral ions after the addition of $\mathrm{Ca}, \mathrm{Pi}$, and $\mathrm{F}$ supplements [23], this increase was not observed for the biofilm fluid, which might suggest the existence of equilibrium or a homeostatic mechanism that maintains ion concentration in the biofilm fluid, regardless of its concentration in the whole biofilm, as previously hypothesized [20].

For samples collected $12 \mathrm{~h}$ after brushing, no significant differences were noted among the dentifrices, indicating that all fluoride retained in the biofilm after brushing was slowly released over time, returning to baseline levels afterwards. This result is consistent with previous data showing higher $\mathrm{F}$ concentrations in whole biofilm samples $1 \mathrm{~h}$ after brushing with $\mathrm{F}$ dentifrices, which returned to baseline (placebo) values $12 \mathrm{~h}$ after the use of a conventional toothpaste [24]. Also, fluoride concentrations in saliva and dental biofilm collected $8 \mathrm{~h}$ after the last use of fluoride products (dentifrices and fluoride solution) did not differ among treatments (placebo, $1100 \mathrm{ppm} \mathrm{F}$, and 1100 ppm F + fluoride solution), suggesting that $\mathrm{F}$ products for home-use have no long-term effect on fluoride concentrations in saliva and in dental biofilm, mainly in residents of an area with a fluoridated water supply $[25,26]$.

Although it is not possible to make a direct comparison between whole biofilm $\mathrm{F}$ concentrations after the use of LFDs supplemented with CaGP [4] or TMP [13] with the present results in the biofilm fluid, the discrepancies between the present results and the abovementioned studies are evident. The only methodological difference in the study protocol was the mode of application of the dentifrices. While a dentifrice:water slurry (1:3) was dripped ex vivo directly on enamel blocks during $1 \mathrm{~min}$ [4], in the present study, volunteers were instructed to brush their teeth with the device in the oral cavity in order to produce a natural dentifrice:saliva slurry, which was later swished in the oral cavity during $30 \mathrm{~s}$. Given that penetration of fluoride into biofilms is highly dependent on biofilm's thickness [27] and that fluoride is mainly restricted to the biofilm/saliva interface after brushing with conventional or low-fluoride toothpastes in thick biofilms [28] (as in the present study), it is likely that the mode of application (dentifrice foam versus dentifrice slurry) and exposure time ( 30 versus $60 \mathrm{~s}$ ) might have played a major role in the results obtained in the present study. This aspect is of outermost relevance and raises questions on the in situ models currently used to assess the effects of fluoride toothpastes on the dynamics of dental caries.

Another considerable aspect was the timing of sample collection. Biofilm samples were collected $60 \mathrm{~min}$ after the treatment with the toothpastes and $55 \mathrm{~min}$ after the cariogenic challenge. It is known that the decline in biofilm fluid $\mathrm{pH}$ to levels lower than 5 following exposure to sucrose lasts about 20 min [29, 30], during which $\mathrm{F}$ concentrations in the biofilm fluid increases due to the dissolution of $\mathrm{CaF}_{2}$ and the release of $\mathrm{F}$ bound to sites on bacteria $[16,20]$. After $40 \mathrm{~min}$, biofilm fluid $\mathrm{pH}$ then rises to 6 or more due to the clearance of sucrose by salivary flow and buffering of hydrogen ions, and this increase is accompanied by a decrease in biofilm fluid $\mathrm{F}$ concentration due to the formation and precipitation of the relatively insoluble $\mathrm{F}$ salts and binding on bacteria. Therefore, performing a cariogenic challenge immediately after the use of the toothpastes ( $60 \mathrm{~min}$ collection) is likely to have affected the results, so that any possible influence of the test toothpastes on biofilm fluid $\mathrm{F}$ levels could not be verified. In this sense, it is noteworthy that the high intensity of the cariogenic challenge used in the present study (30\% sucrose solution) might have produced an intense depleting effect on fluoride levels in the dental biofilm. As for $\mathrm{Ca}$ concentrations in the biofilm fluid, no significant differences were observed for time after brushing. Surprisingly, the highest values of $\mathrm{Ca}$ concentration were obtained for placebo and 550F-CaGP, lowest were found for 550F-TMP and 1100. The reasons for this discrepancy are not known. However, considering that $\mathrm{F}$ is retained on the biofilm mediated by $\mathrm{Ca}$ bindings on bacteria walls [15], it is possible that brushing the teeth with a conventional dentifrice (1100 ppm) allowed $\mathrm{F}$ binding to Ca present in the biomass (mainly bacterial surfaces), further allowing ionic $\mathrm{Ca}$ present in biofilm fluid to bind to the biomass as well, which would, in turn, reduce $\mathrm{Ca}$ concentrations in the biofilm fluid. On the other hand, as placebo has no fluoride, the abovementioned mechanism would not occur, so that ionic $\mathrm{Ca}$ would remain free in the fluid phase of the biofilm. Moreover, the high values found for the 550F-CaGP dentifrice suggest that $\mathrm{CaGP}$ can be considered as a source of free calcium [31], what may help to explain the anticaries effect of this toothpaste in caries progression in children [7].

This investigation provided additional information for better understanding of the mechanisms of $\mathrm{F}$ and $\mathrm{Ca}$ 
uptake by biofilm fluid. In this sense, the lack of synergistic effect between $\mathrm{F}$ and TMP or CaGP in the biofilm fluid seen in the present study along with previous in vitro and in situ data indicates that the anticaries effects of LFDs supplemented with these salts may be more related to the interaction of these salts with tooth enamel than with an increased availability of $\mathrm{F}$ and $\mathrm{Ca}$ ions in the biofilm fluid. Further studies should be carried out to complement this in situ investigation, in order to clearly verify the clinical benefits provided by the supplementation of LFDs with phosphate salts, as well as to provide stronger evidence on the mechanisms of action of toothpastes containing TMP or CaGP.

Acknowledgments The authors thank to the volunteers for taking part in the study. This study was supported by CAPES (scholarship to the first author) and CNPq (Grant \#479727/2011-2), which played no role in the study design, data collection and analysis, decision to publish or preparation of the manuscript. The second author holds a patent request for a product used in the study, by the National Institute of Industrial Property, INPI/SP, on 04/29/2008 under number 018080026091, PI0801811-1, and published on January 11, 2011.

Compliance with ethical standards All procedures performed in studies involving human participants were in accordance with the ethical standards of the institutional and/or national research committee and with the 1964 Helsinki Declaration and its later amendments or comparable ethical standards.

Funding This study was funded by CAPES (scholarship to the first author) and CNPq (grant \#479,727/2011-2).

Conflict of interest Ms. Nagata received a scholarship from CAPESBrazil. Prof. Delbem received a grand from CNPq (479727/2011-2) and holds a patent request for a product used in the study, by the National Institute of Industrial Property, INPI/SP, on 04/29/2008 under number 018080026091, PI0801811-1, and published on January 11, 2011.

Informed consent Informed consent was obtained from all individual participants included in the study.

\section{References}

1. Walsh T, Worthington HV, Glenny AM, Appelbe P, Marinho VC, Shi X (2010) Fluoride toothpastes of different concentrations for preventing dental caries in children and adolescents. Cochrane Database Syst Rev 20:CD007868

2. Wong MC, Clarkson J, Glenny AM, Lo EC, Marinho VC, Tsang BW, Walsh T, Worthington HV (2011) Cochrane reviews on the benefits/risks of fluoride toothpastes. J Dent Res 90:573-579

3. Pessan JP, Toumba KJ, Buzalaf MA (2011) Topical use of fluorides for caries control. Monogr Oral Sci 22:115-132

4. Amaral JG, Sassaki KT, Martinhon CCR, Delbem ACB (2013) Effect of low-fluoride dentifrices supplemented with calcium glycerophosphate on enamel demineralization in situ. Am J Dent 26: 75-80
5. Zaze ACSF, Dias AP, Amaral JG, Miyasaki ML, Sassaki KT, Delbem ACB (2014) In situ evaluation of low- fluoride toothpastes associated to calcium glycerophosphate on enamel remineralization. J Dent 42:1621-1625

6. Takeshita EM, Castro LP, Sassaki KT, Delbem ACB (2009) In vitro evaluation with low fluoride content supplemented with trimetaphosphate. Caries Res 43:50-56

7. Freire IR, Pessan JP, Amaral JG, Martinhon CC, Cunha RF, Delbem ACB (2016) Anticaries effect of low-fluoride dentifrices with phosphates in children: A randomized, controlled trial. J Dent. doi:10.1016/j.jdent.2016.04.013

8. Danelon M, Takeshita EM, Peixoto LC, Sassaki KT, Delbem ACB (2014) Effect of fluoride gels supplemented with sodium trimetaphosphate in reducing demineralization. Clin Oral Investig 18:1119-1127

9. Pancote LP, Manarelli MM, Danelon M, Delbem ACB (2014) Effect of fluoride gels supplemented with sodium trimetaphosphate on enamel erosion and abrasion: in vitro study. Arch Oral Biol 59: 336-340

10. Manarelli MM, Delbem ACB, Lima TMT, Castilho FCN, Pessan JP (2014) In vitro remineralizing effect of fluoride varnishes containing sodium trimetaphosphate. Caries Res 48:299-305

11. Moretto MJ, Delbem ACB, Manarelli MM, Pessan JP, Martinhon CCR (2013) Effect of fluoride varnish supplemented with sodium trimetaphosphate on enamel erosion and abrasion: an in situ/ex vivo study. J Dent 41:1302-1306

12. Zaze ACSF, Dias AP, Sassaki KT, Delbem ACB (2014) The effects of low-fluoride toothpaste supplemented with calcium glycerophosphate on enamel demineralization. Clin Oral Investig 18:16191624

13. Takeshita EM, Danelon M, Castro LP, Sassaki KT, Delbem ACB (2015) Effectiveness of a toothpaste with low fluoride content combined with trimetaphosphate on dental biofilm and enamel demineralization in situ. Caries Res 49:394-400

14. Kaufman HW, Kleinberg I (1973) X-ray diffraction examination of calcium phosphate in dental plaque. Calcif Tissue Res 11:97-104

15. Rose RK, Shellis RP, Lee AR (1996) The role of cation bridging in microbial fluoride binding. Caries Res 30:458-464

16. Gao XJ, Fan Y, Kent RL Jr, Van Houte J, Margolis HC (2001) Association of caries activity with the composition of dental plaque fluid. J Dent Res 80:1834-1839

17. Tenuta LM, Zamataro CB, Del Bel Cury AA, Tabchoury CP, Cury JA (2009) Mechanism of fluoride dentifrice effect on enamel demineralization. Caries Res 43:278-285

18. Delbem AC, Cury JA (2002) Effect of application time of APF and $\mathrm{NaF}$ gels on microhardness and fluoride uptake of in vitro enamel caries. Am J Dent 15:169-172

19. Vogel GL, Mao Y, Carey CM, Chow LC (1997) Increased overnight fluoride concentrations in saliva, plaque, and plaque fluid after a novel two-solution rinse. J Dent Res 76:761-767

20. Tenuta LM, Del Bel Cury AA, Bortolin MC, Vogel GL, Cury JA (2006) $\mathrm{Ca}$, pi, and $\mathrm{F}$ in the fluid of biofilm formed under sucrose. $\mathrm{J}$ Dent Res 85:834-838

21. Favretto CO, Danelon M, Castilho FCN, Vieira AEM, Delbem ACB (2013) In vitro evaluation of the effect of mouth rinse with trimetaphosphate on enamel demineralization. Caries Res 47:532538

22. Tenuta LM, Cenci MS, Cury AA, Pereira-Cenci T, Tabchoury CP, Moi GP, Cury JA (2009) Effect of a calcium glycerophosphate fluoride dentifrice formulation on enamel demineralization in situ. Am J Dent 22:278-282

23. Pearce EIF, Margolis HC, Kent RL Jr (1999) Effect of in situ plaque mineral supplementation on the state of saturation of plaque fluid during sugar-induced acidogenesis. Eur J Oral Sci 107:251-259 
24. Whitford GM, Wasdin JL, Schafer TE, Adair SM (2002) Plaque fluoride concentrations are dependent on plaque calcium concentrations. Caries Res 36:256-265

25. Souza DCC, Maltz M, Hashizume LN (2014) Fluoride retention in saliva and in dental biofilm after different home-use fluoride treatments. Braz Oral Res 28:1-5

26. Kondo KY, Buzalaf MAR, Manarelli MM, Delbem ACB, Pessan JP (2015). Effects of pH and fluoride concentration of dentifrices on fluoride levels in saliva, biofilm and biofilm fluid in vivo. Clin Oral Investig (in press).

27. Watson PS, Pontefract HA, Devine DA, Shore RC, Nattress BR (2005) Kirkham J et al. Penetration of fluoride into natural plaque biofilmsJ Dent Res 84:451-455
28. Pessan JP, Alves KM, Italiani Fde M, Ramires I, Lauris JRP, Whitford GM, Toumba KJ, Robinson C, Buzalaf MAR (2014) Distribution of fluoride and calcium in plaque biofilms after the use of conventional and low-fluoride dentifrices. Int J Paediatr Dent 24:293-302

29. Stephan RM, Miller BF (1943) A quantitative method for evaluating physical and chemical agents which modify production of acids in bacterial plaques on human teeth. J Dent Res 22:45-51

30. Buzalaf MA, Pessan JP, Honorio HM, ten Cate JM (2011) Mechanisms of action of fluoride for caries control. Monogr Oral Sci 22:97-114

31. Lynch RJM (2004) Calcium glycerophosphate and caries: a review of the literature. Int Dent J 54:310-314 